\title{
Analysis of the Virulence Modulating Region of Potato Spindle Tuber Viroid (PSTVd) by Site-Directed Mutagenesis
}

\author{
ROSEMARIE W. HAMMOND \\ Microbiology and Plant Pathology Laboratory, Plant Sciences Insilute, USDA-ARS, Beltsville, Maryland 20705 \\ Received October 30, 1991; accepted December 26, 1991
}

\begin{abstract}
A series of nucleotide substitutions $\left(\mathrm{G}_{46} \rightarrow \mathrm{C} ; \mathrm{C}_{47} \rightarrow A ; \mathrm{C}_{315} \rightarrow \mathrm{U} ; \mathrm{U}_{317} \rightarrow \mathrm{C}\right)$ were introduced into the virulence modulating region of the intermediate strain of potato spindle tuber viroid (PSTVd) in order to examine their effect upon viroid infectivity and pathogenicity with the presence of all four mutations resulting in the sequence of a previously reported severe strain of PSTVd. Eight of the resulting mutant cDNAs were characterized for infectivity and symptom induction in tomato, and the secondary structure of their corresponding RNAs was examined. The combined results of infectivity, computer analysis, and chemical mapping data imply that a previously proposed correlation between thermodynamic stability and PSTVd pathogenicity does not hold true in all cases and suggest that conformation and/or sequence-specific interactions with host factors play a role in symptom development. (c) 1992 Academic Press, Inc.
\end{abstract}

\section{INTRODUCTION}

Viroids are the smallest known pathogenic agents of plants and are distinguished from viruses by their lack of a protein coat and lack of a protein coding capacity. Viroid pathogenicity in infected plants is a complex process that is influenced by both the viroid and the host plant genomes, e.g., infections by different viroid strains in the same host or alternatively by the same viroid strain in different hosts may be latent or result in symptoms ranging from mild to severe in intensity.

As viroids replicate and exert pathogenic effects without encoding proteins, the implication is that viroid functions are mediated through sequence and structural signals alone. One approach to investigating the relationship between structure and function involves comparative sequence analysis of different viroid species (Flores, 1984; Keese and Symons, 1985) and of naturally occurring strains of the same viroid (Schnölzer et al., 1985; Visvader and Symons, 1985, 1986). Comparisons of potato spindle tuber viroid (PSTVd) (Schnölzer et al. 1985) and citrus exocortis viroid (CEVd) (Visvader and Symons, 1985) field isolates whose symptoms in tomato vary widely suggest that pathogenicity modulating functions are localized within two regions of the native structure-the socalled pathogenicity $(P)$ domain, a term which was coined for all the viroids examined by Keese and Symons (1985) [or the virulence modulating (VM) region, a term coined for PSTVd (Schnölzer et al., 1985)] and the variable $(V)$ domain. In addition, Shikata (1990) reported that sequence variations of hop stunt viroid (HSVd) isolates are confined to a region corresponding to the P domain of PSTVd and CEVd.
Four earlier reports have dealt with the association of the $\mathrm{P}$ domain with pathogenicity. Schnölzer et al. (1985) reported that the pathogenicity of PSTVd isolates which produce mild to lethal symptoms in tomato could be correlated with nucleotide differences within the $\mathrm{VM}$ region with boundaries of $\mathrm{nt} 42-60$ and $\mathrm{nt}$ 300-319 (see Fig. 1). This region includes an area of calculated thermal instability, the premelting (PM) loop 1 (Steger et al., 1984) (Fig. 1). Calculations of the thermodynamic stability of the VM region between the naturally occurring isolates suggested that stability could be inversely correlated with an increase in symptom severity in tomato.

In the second report, the ability to incite mild and severe disease symptoms could be assigned to two distinct classes of CEVd sequences, which differ primarily in the sequence composition of the $\mathrm{P}$ domain (Visvader and Symons, 1985). Subsequent infectivity studies with chimeric cDNA clones derived from mild and severe strains of CEVd have shown that sequence differences correlated with symptom severity are located in the $P$ domain (Visvader and Symons, 1986). Symptom severity, however, could not always be correlated with the calculated stability of this domain. In direct contrast to the results obtained with PSTVd, Visvader and Symons (1985) found the calculated stability of the premelting region to be considerably higher in severe strains than in those expressing mild symptoms. Finally, Owens (1990) examined the effect of a series of nucleotide substitutions in the right side of the $P$ domain of tomato apical stunt viroid (TASVd). None of the alterations appeared to have an effect upon symptom expression, although rearrangements of the RNA secondary structure were predicted to occur. 


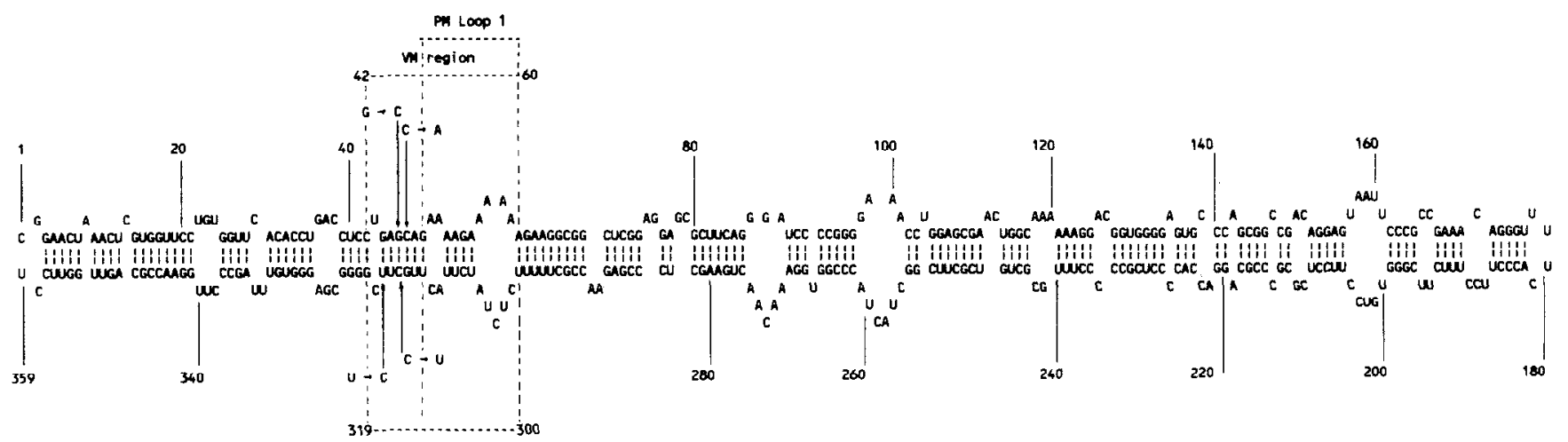

FIG. 1. Schematic diagram of the intermediate strain of PSTVd (PSTVd-B5) as reported by Schnölzer et al. (1985). The VM and PM loop 1 are described in the text. The four nucleotide differences between the intermediate and a severe strain [KF440-1, Schnölzer et al. (1985)] are shown. The numbering designates the position of nucleotides in the intermediate strain of PSTVd (PSTVd-B5).

To systematically address the question of pathogenicity in PSTVd, a series of site-directed mutations have been introduced into the VM region of the intermediate strain of PSTVd. Sequence comparisons between the intermediate (Type) and a severe to lethal strain (KF440-1) of PSTVd have previously shown that only four nucleotide changes occur in this region (see Fig. 1) (Sänger, 1982; Schnölzer et al., 1985). To test the "plasticity" of this region, we had previously introduced one of these changes, a single $\mathrm{G} \rightarrow \mathrm{C}$ transversion at residue 46 (Hammond and Owens, 1987). This mutation resulted in complete loss of infectivity of the PSTVd cDNA.

This report describes the introduction of single and multiple mutations to create various combinations of the four nucleotide differences between these two PSTVd strains. Specifically, the questions being addressed in this mutational study are: (a) do artificially introduced mutations in the $V M$ region affect virulence and/or infectivity of PSTVd cDNAs and how? and (b) what are the common and unique structural features of the corresponding variant RNAs? The combined biological and biochemical data presented here demonstrate that thermodynamic stability in the VM region in PSTVd is not the sole contributing factor to the pathogenicity of PSTVd in tomato.

\section{MATERIALS AND METHODS}

\section{Bacteria, enzymes}

Escherichia coli strains JM83, JM107, and GM119 (dam, supE44, $\mathrm{F}^{\prime} \mathrm{Km}^{\mathrm{R}}$ ) were used to propagate recombinant phage and plasmids. Bacteria were grown in minimal media or yeast-tryptone broth supplemented with ampicillin at $25-50 \mu \mathrm{g} / \mathrm{ml}$ or kanamycin at $100 \mu \mathrm{g} / \mathrm{ml}$, as appropriate (Maniatis, 1982). Enzymes were obtained from New England Biolabs (Beverly, MA
01915), ${ }^{1}$ Boehringer-Mannheim (Indianapolis, IN 46250), GIBCO-BRL Life Technologies (Gaithersburg, MD 20877), and Promega Corp. (Madison, WI 53711) and were used in accordance with suppliers' instructions.

\section{Oligonucleotide-directed mutagenesis}

PSTVd mutants were constructed using synthetic oligonucleotides and a single-stranded $\mathrm{M} 13$ template, as described (Hammond and Owens, 1987). Many oligonucleotides were synthesized on an Applied Biosystems 380A DNA synthesizer (Foster City, CA 94403) and were purified by HPLC; others were purchased from Synthecell (Rockville, MD 20850). The primers used for oligonucleotide mutagenesis were: PSTV-D = $5^{\prime}$ TTITCTGGTCAGGAGGT 3'; PSTV-M1 $=5^{\prime}$ TCTTICTIGTCAGGAGG 3'; PSTV-M2 = 5' GCCCCGGAACAAGTAAG $3^{\prime} ;$ and PSTV-M3 $=5^{\prime}$ CCTCGCCCCGGAACAAGTAAGA $3{ }^{\prime}$, where the base changes are underlined. The replicative (RF) and single-stranded forms of bacteriophage $\mathrm{M} 13$ were prepared from infected JM107 or phage particles (Messing, 1983).

Following isolation and characterization of a mutant cDNA, the PSTVd insert was subcloned into the BamHI site of pUC9 or pSP64, and intact plasmid DNA or RNA transcripts were used for infectivity studies. Small scale plasmid DNA preparations were isolated by the alkaline lysis method (Maniatis et al., 1982) and large scale pUC9 or pSP64 DNA preparations were made following protocols provided by Promega Corp.

\footnotetext{
${ }^{1}$ Mention of trademark, proprietary product or vendor does not constitute a guarantee or warranty of the product by the U.S. Department of Agriculture and does not imply its approval to the exclusion of other products or vendors that may be suitable.
} 


\section{Sequence analysis}

Double-stranded plasmid or RF DNAs were sequenced using $\left[{ }^{32} \mathrm{P}\right] \mathrm{dATP}$ and a modified chain termination method for dsDNA templates (Kormeluk et al., 1985). Gel-purified single-stranded viroid RNA was used as a template for dideoxy sequence analysis (Zimmern and Kaesberg, 1978; as described in Hammond et al., 1989) using oligonucleotide primers PSTV-T2 $=5^{\prime}$ CTGTTCGGCGGGAATTA $3^{\prime}$ (complementary to nt 157-174 of PSTVd) and RF2 $=5^{\prime}$ GGCTTCAGTTGTTCCACC $3^{\prime}$ (complementary to $\mathrm{nt}$ 265-283 of PSTVd).

\section{Infectivity assays}

Infectivity of recombinant DNA plasmids containing PSTVd inserts was performed as previously described (Hammond and Owens, 1987). Isolation and hybridization analysis of tomato low molecular weight (LMW) RNA (RNA soluble in $2 \mathrm{MLiCl}$ ) have been described (Owens et al., 1986). Circular viroid RNAs were purified as described (Hammond et al., 1989).

\section{Preparation of RNAs for structural mapping}

RNAs were synthesized by in vitro transcription of pSP64 plasmid DNAs harboring the various PSTVd mutants described in the text. The PSTVd cDNAs were inserted into the unique $\mathrm{BamHI}$ site of the multiple cloning site (MCS) in the plasmid. Prior to transcription, the plasmids were linearized by restriction digestion with Pvull, which cleaves the DNA 232 bp downstream of the MCS. Transcription reactions contained $40 \mathrm{mM}$ Tris- $\mathrm{HCl}, \mathrm{pH} 7.9,6 \mathrm{mM} \mathrm{MgCl}$, $2 \mathrm{mM}$ spermidine, 0.5 mM each of rATP, rCTP, rGTP, and UTP, $1 \mathrm{mMDTT}, 1$ unit $/ \mu$ l RNasin, 5 units of SP6 polymerase, and $1 \mu \mathrm{g}$ of linearized DNA template. The reactions were incubated at $37^{\circ}$ for $30 \mathrm{~min}$, followed by phenol/chloroform extraction. Total nucleic acids were precipitated in the presence of sodium acetate and ethanol. Aliquots of the transcription reactions were analyzed by electrophoresis through $5 \%$ polyacrylamide gels containing 8 $M$ urea and $1 \times$ TBE buffer.

\section{Chemical modification and sequence analysis of synthetic RNAs}

The general procedures described by Mougel et al. (1987) were followed. Prior to modification, 1 to $2 \mu \mathrm{g}$ of each transcript RNA was heated to $65^{\circ}$ and annealed at room temperature $\left(25^{\circ}\right)$ under conditions where monomeric linear viroid RNAs assume their thermodynamically most stable structure (in a buffer containing $50 \mathrm{~m} M$ sodium cacodylate, $\mathrm{pH} 6.8,1 \mathrm{mM}$ EDTA). For modification of the RNA, $1 \mu$ l of dimethyl sulfate (DMS,
Aldrich Chemical CO., Inc. Milwaukee, WI 53233) was added to a $100-\mu l$ solution containing the RNA in 50 $\mathrm{mM}$ sodium cacodylate, $\mathrm{pH} 6.8,20 \mathrm{mM} \mathrm{Mg}$ acetate. The reactions were incubated at room temperature for $5 \mathrm{~min}$, unless otherwise noted. Nucleic acids were precipitated in the presence of sodium acetate and ethanol and $5 \mu \mathrm{g}$ yeast tRNA as carrier. Following a $70 \%$ ethanol wash of the nucleic acid pellets, the RNAs were sequenced using primer extension with synthetic oligonucleotides as described above. Briefly, oligonucleotide primers, primer $1=5^{\prime}$ CAACTGCGGTTCCA $3^{\prime}$ (complementary to nt 340-353 in PSTVd) and primer 2 $=5^{\prime}$ GACCATGATTACGAATTC $3^{\prime}$ (complementary to nt 52-69 of pSP64), were used in the sequence reactions. ${ }^{32} \mathrm{P}$-labeled primer (3.2 pmol) (McGraw, 1984) was annealed to the transcript RNA and the RNA was sequenced as described (Hammond et al., 1989). The reaction products were fractionated in $8 \%$ polyacrylamide gels containing $1 \times$ TBE $(89 \mathrm{~m} M$ Tris, $\mathrm{pH} 8.3,89$ $\mathrm{mM}$ boric acid, $2.5 \mathrm{mM}$ EDTA) and $7 M$ urea.

\section{Computer analysis}

Secondary structures for the mutant and wild-type RNAs were calculated on the basis of their nucleotide sequence and their free energy parameters using the computer program of Zuker (1989) running on a Digital Al VAX Station (Digital Equipment Corp., Maynard, MA). Nucleotides $30-83$ and 279-331 of PSTVd, which includes the pathogenicity modulating region, were entered into the computer. No restrictions on base-pairing were introduced into the analysis. To simulate the actual thermodynamic melting behavior of a closed circular molecule, the $5^{\prime}$ end of the helical region was allowed to pair and a 4-nucleotide loop was introduced into the $3^{\prime}$ end, adjacent to a helical region.

\section{RESULTS}

\section{Constructions and infectivity assays}

Oligonucleotide-directed mutagenesis was used to introduce single or multiple changes into the VM region of the intermediate strain of PSTVd (PSTVd-B5) (Table 1). Seven mutant cDNAs in addition to the unmodified PSTVd-B5 were compared for infectivity and severity of symptom production. Intact, full-length PSTVd monomeric cDNAs in pUC9 were tested for infectivity (Table 2). These cDNAs contain an 11-nucleotide terminal repeat $\left(5^{\prime}\right.$ GGATCCCCGGG $\left.3^{\prime}\right)$ to maximize their specific infectivity (Tabler and Sänger, 1984). PSTVd-B5 induces intermediate-type symptoms 3-4 weeks following inoculation of the cotyledons of 6- to 7-day-old tomato (cv. Rutgers) seedlings (Fig. 2A). 
TABLE 1

Site-Directed MutATIONS INTRODUCED INTO PSTVD-B5

\begin{tabular}{lccr}
\hline Plasmid & Mutations & Plasmid & Mutations \\
\hline PSTVd-B5 & Wild-type & PSTVd-M3/D & $\mathrm{G}_{46} \rightarrow \mathrm{C}$ \\
& & & $\mathrm{C}_{316} \rightarrow \mathrm{U}$ \\
& & $\mathrm{U}_{317} \rightarrow \mathrm{C}$ \\
PSTVd-D & $\mathrm{G}_{46} \rightarrow \mathrm{C}$ & PSTVd-M3/4 & $\mathrm{C}_{315} \rightarrow \mathrm{U}$ \\
& & & $U_{317} \rightarrow \mathrm{C}$ \\
PSTVd-M1 & $\mathrm{G}_{46} \rightarrow \mathrm{C}$ & PSTVd-M2/M1 & $\mathrm{G}_{46} \rightarrow \mathrm{C}$ \\
& $\mathrm{C}_{47} \rightarrow \mathrm{A}$ & & $\mathrm{C}_{47} \rightarrow \mathrm{A}$ \\
& & & $\mathrm{C}_{315} \rightarrow U$ \\
PSTVd-M2 & $\mathrm{G}_{46} \rightarrow \mathrm{C}$ & PSTVd-M3/M1 & $\mathrm{G}_{46} \rightarrow \mathrm{C}$ \\
& $\mathrm{C}_{315} \rightarrow \mathrm{U}$ & & $\mathrm{C}_{47} \rightarrow \mathrm{A}$ \\
& & & $\mathrm{C}_{315} \rightarrow \mathrm{U}$ \\
& & & $\mathrm{U}_{317} \rightarrow \mathrm{C}$ \\
\hline
\end{tabular}

PSTVd-D, a noninfectious cDNA containing a single $G \rightarrow C$ transversion at position 46 was reported previously (Hammond and Owens, 1987). Introduction of an additional $\mathrm{C} \rightarrow \mathrm{A}$ transversion at residue 47 restores infectivity and induces intermediate-type symptoms (Fig. 2A). A cDNA containing a third mutation, a $\mathrm{C} \rightarrow \mathrm{U}$ transition at position 315, produced PSTVd-M2/M1. Like PSTVd-M1, this construct was also infectious and produced intermediate-type symptoms. PSTVd-M3/ M1 contains all four mutations present in a naturally occurring lethal strain (i.e., $\mathrm{G}_{46} \rightarrow \mathrm{C}, \mathrm{C}_{47} \rightarrow \mathrm{A}, \mathrm{C}_{315} \rightarrow$ $U, U_{317} \rightarrow C$ ) and caused severe symptoms (Fig. 2A).

Three additional mutants, all of which were noninfectious as determined by dot-blot hybridization, were also constructed (PSTVd-M2, PSTVd-M3/D, and PSTVd-M3/4) (Tables 1 and 2).

\section{Further characterization of infectious mutants}

Although the symptoms expressed by the viable mutants ranged from intermediate to severe in intensity (Fig. 2A), the viroid titer in the upper leaves collected from single plants 6 weeks postinoculation was not significantly different among the variants, although there was some plant to plant variability (e.g., PSTVdB5) (Fig. 2B). However, viroid-related progeny and symptoms appeared earlier in plants inoculated with PSTVd-M3/M1 than in those plants inoculated with PSTVd-B5, PSTVd-M1, or PSTVd-M2/M1 cDNAs (Table 2). Passaging of equal amounts of progeny to a second set of plants, following purification and estimation of viroid titer from the first set of inoculated plants, resulted in similar observations (data not shown). Nu-

TABLE 2

BIOLOGICAL AND BIOPHYSICAL PROPERTIES OF MUTANT PSTVd CDNAS

\begin{tabular}{|c|c|c|c|c|c|c|}
\hline \multirow[b]{2}{*}{ Plasmid } & \multirow[b]{2}{*}{ D.P.I. ${ }^{a}$} & \multicolumn{3}{|c|}{ Infectivity ${ }^{b}$ ( $\mu \mathrm{g}$ DNA/plant) } & \multirow[b]{2}{*}{ Symptoms } & \multirow{2}{*}{$\begin{array}{c}\text { Free energy } \\
\Delta(\Delta G)(\mathrm{kcal} / \mathrm{mol})\end{array}$} \\
\hline & & 2.0 & 0.2 & 0.02 & & \\
\hline \multirow[t]{2}{*}{ PSTVd-B5 } & 14 & $4 / 10$ & $0 / 10$ & $0 / 10$ & & \\
\hline & 28 & $10 / 10$ & $4 / 10$ & $0 / 10$ & Intermediate & 0 \\
\hline \multirow[t]{2}{*}{ PSTVd-D } & 14 & $0 / 10$ & $N T$ & NT & & \\
\hline & 28 & $0 / 10$ & NT & NT & - & +4.8 \\
\hline \multirow{2}{*}{ PSTVd-M1 } & 14 & $4 / 10$ & $0 / 10$ & $0 / 10$ & & \\
\hline & 28 & $10 / 10$ & $4 / 10$ & $1 / 10$ & Intermediate & +2.1 \\
\hline \multirow[t]{2}{*}{ PSTVd-M2 } & 14 & $0 / 10$ & NT & NT & & \\
\hline & 28 & $0 / 10$ & NT & NT & - & +4.6 \\
\hline \multirow[t]{2}{*}{ PSTVd-M3/D } & 14 & $0 / 10$ & NT & NT & & \\
\hline & 28 & $0 / 10$ & NT & NT & $\ldots$ & +6.5 \\
\hline \multirow[t]{2}{*}{ PSTVd-M $3 / 4$} & 14 & $0 / 10$ & NT & NT & & \\
\hline & 28 & $0 / 10$ & NT & NT & - & +1.5 \\
\hline \multirow[t]{2}{*}{ PSTVd-M2/M1 } & 14 & $4 / 10$ & $1 / 10$ & $0 / 10$ & & \\
\hline & 28 & $10 / 10$ & $4 / 10$ & $1 / 10$ & Intermediate & +2.1 \\
\hline \multirow{2}{*}{ PSTVd-M3/M1 } & 14 & $8 / 10$ & $4 / 10$ & $0 / 10$ & & \\
\hline & 28 & $10 / 10$ & $8 / 10$ & $2 / 10$ & Severe & +2.3 \\
\hline
\end{tabular}

${ }^{\circ}$ D.P.I., days postinoculation.

${ }^{b}$ Infectivity was monitored by nucleic acid hybridization of "Rutgers" tomato plants at 2 to 4 weeks following inoculation of cotyledons with intact plasmid DNA as described (Materials and Methods) and is expressed as numbers of plants infected per total number of plants inoculated. NT, not tested.

$c$ The most stable thermodynamic structure for the region nt 29-73 and nt 285-331 was calculated using the algorithm of Zuker (1989) as described (Materials and Methods). I he change $[\Delta(\Delta G)]$ in free energy value is normalized to the free energy value obtained for the most stable structure of the intermediate strain. $A(+)$ value signifies a decrease in the thermodynamic stability of the region. 

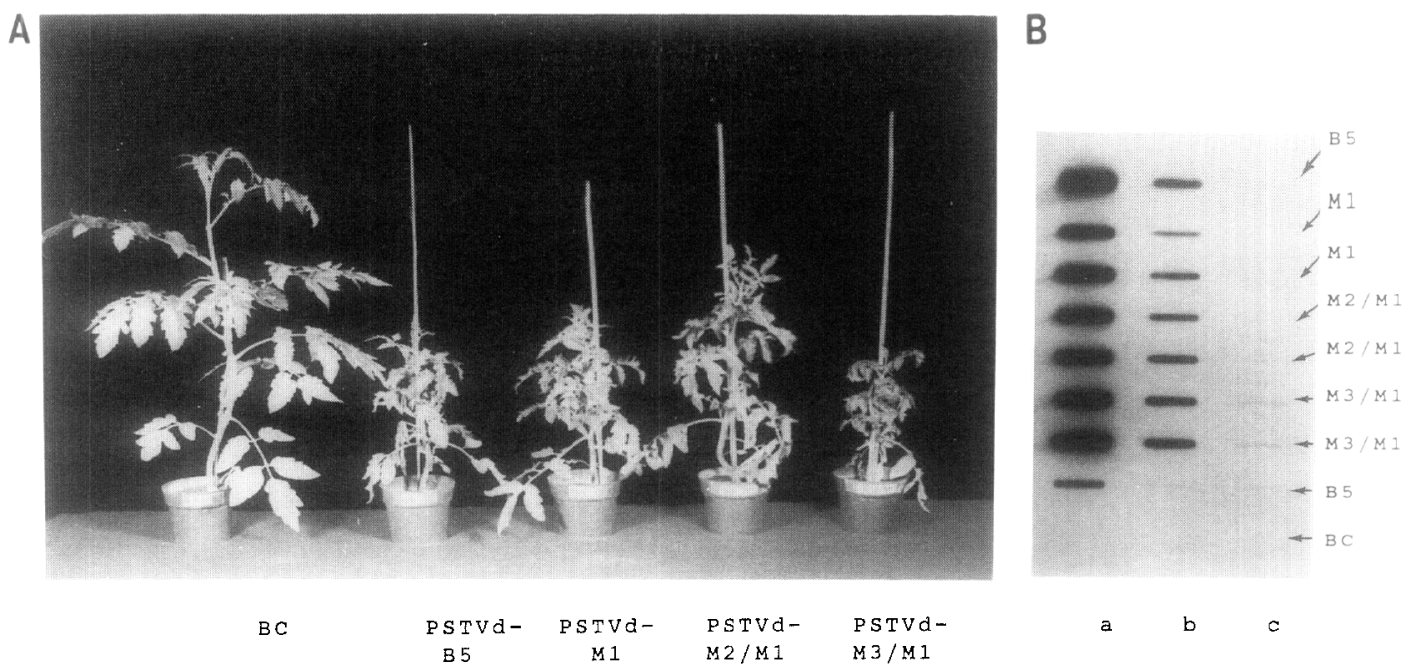

FIG. 2. Biological properties of infectious PSTVd mutants. (A) Symptom production on "Rutgers" tomato plants examined at 6 weeks following inoculation with PSTVd mutants as described in the text. These plants were inoculated with $2 \mu \mathrm{g}$ plasmid DNA per plant. (B) Viroid titer in representative tomato plants inoculated with the PSTVd mutants shown in A. Apical leaf tissue (50 mg) was extracted from plants at 6 weeks postinoculation and aliquots were applied in 10-fold dilutions $(\mathrm{a}-\mathrm{c})$ to a nitrocellulose membrane. The membrane was then hybridized with a PSTVd SP6-generated ${ }^{32}$ P-labeled RNA probe. BC, buffer control.

cleic acid hybridization analysis using oligonucleotide probes, as well as direct sequence analysis of the progeny isolated from plants inoculated with the mutant cDNAs, revealed no obvious sequence heterogeneity or sequence reversions (data not shown). These analyses will only detect sequence heterogeneities if they are a significant proportion of the population. The absence of readily detectable sequence variations suggests that the mutations introduced into the viroid RNAs are stable in tomato.

\section{Structural analysis of PSTVd RNAs}

Computer analyses. The primary sequence data were analyzed and the free energies of the most stable secondary structures of the P domains of the mutant RNAs were calculated as described by Zuker (1989) and are shown in Table 2. In most cases, more than one structure was possible at the lowest free energy value. A representative example of each of the infectious variants is shown in Fig. 3. In all cases, including the noninfectious variants (data not shown), the characteristic stem and loop structure of viroid RNAs was obtained (Langowski et al., 1978); however, the position of the loops varied with the sequence of the variant.

The free energy $(\Delta G)$ values were calculated at $37^{\circ}$ (Zuker, 1989) and the values obtained for the thermodynamically most stable structure were normalized to that obtained for PSTVd-B5, i.e., the values reported in Table $2[\Delta(\Delta G)]$ reflect the difference in free energy compared to PSTVd-B5. A $(+)$ value signifies a de- crease in the thermodynamic stability. With the exception of PSTVd-M3/4, the P domains of all the noninfectious mutants were markedly destabilized. The infectious mutants were calculated to have $[\Delta(\Delta G)]$ values similar to those of PSIVd-B5 $(+2.1 \pm 2.3$ $\mathrm{kcal} / \mathrm{mol})$.

Chemical reactivity. Because free energy minimization data alone is not sufficient to determine RNA folding with confidence, the conformation of the $\mathrm{VM}$ region in solution was directly examined. The method used involves the chemical modification of accessible (and presumed single-stranded) bases in RNA molecules followed by reverse transcription using a specific primer in the presence of dideoxy nucleotides to determine which bases were modified. Reactivity to the chemical probe was carefully examined and experiments were repeated to ensure that results were reproducible.

In vitro generated RNA transcripts were analyzed as well as progeny from infectious cDNAs. In vitro transcripts were generated using SP6 RNA polymerase, and the mixture was treated with DNase (to remove the plasmid DNA template) prior to analysis (Fig. 4). As described under Materials and Methods, prior to chemical modification, the RNAs were denatured and cooled under conditions which should allow the "native" viroid conformation to form in solution (Steger et al., 1986). Using these conditions, the chemical probing results obtained for transcripts or circular progeny RNA of PSTVd-M3/M1 were similar, therefore most experiments were performed using transcripts as these were easier to obtain in large quantities. 

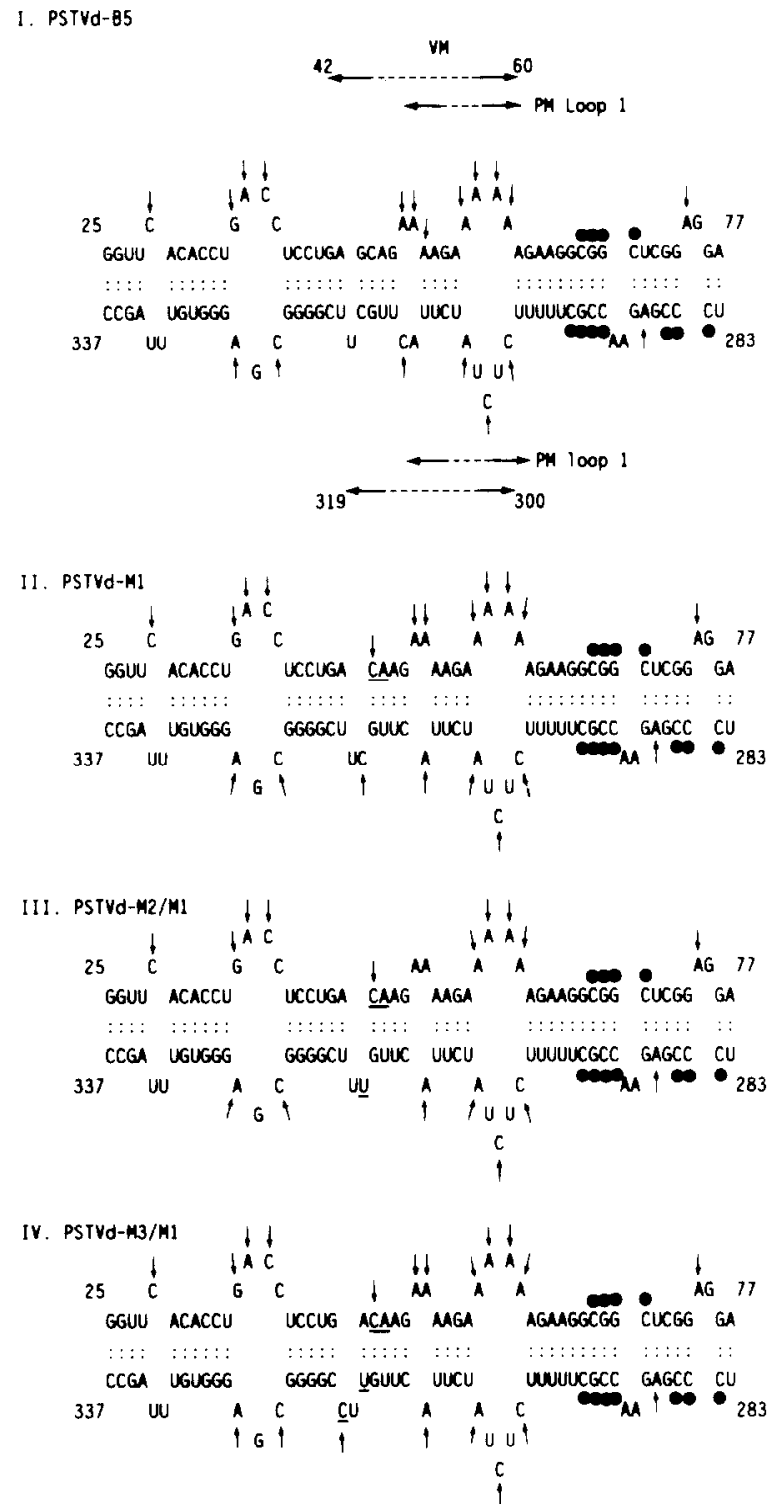

FIG. 3. Predicted structural effects of mutations in the VM region of PSTVd. The most stable secondary structure models were determined using the algorithm of Zuker (1989), as described under Materials and Methods. Where two or more structures were possible for the lowest free energy, a representative example of each is shown. Sites of chemical modification with DMS are superimposed on the secondary structure models. Residues marked with an arrow are adenosines and cytidines which are modified by DMS to a strong or medium extent, indicative of single-strandedness. Solid circles indicate strong stops which are present when the unmodificd RN $\wedge s$ arc sequenced as described. Mutated nucleotides are underlined. (I) PSTVd-B5 (intermediate strain); (II) PSTVd-M1; (III) PSTVd-M2/M1; (IV) PSTVd-M3/M1. Numbering designates the nucleotide position in the PSTVd intermediate strain sequence.

Figure 4 shows representative autoradiographs of some chemical probing experiments. To interpret the results, one assumes that elongation terminates (or pauses) 1 nucleotide before the methylation of a resi- due (Inoue and Cech, 1985). The reactivities of certain residues to DMS were monitored using standard conditions described under Materials and Methods and the results are shown in Fig. 4. Prominent pauses (marked by solid circles) occur at several positions in the untreated transcripts $(-)$ and may reflect the difficulty that reverse transcriptase has in melting helical regions; for example, the $C$ residue at nt 39 (Figs. $4 \mathrm{~A}$ and $4 B$ ) which appears at the $3^{\prime}$ side of a stem structure and $n t$ 65-68 (Figs. 4A and 4B) and nt 292-295 (Fig. $4 C$ ) which compose a highly $G / C$ base-paired region in the native structure (see Fig. 1). These results suggest that the transcripts have assumed the native structure under the annealing conditions chosen.

Dimethyl sulfate methylates the $\mathrm{N}-1$ of adenosine, the N-3 of cytosine, and to a lesser extent the N-7 of guanosine (Inoue and Cech, 1985). The data presented in Fig. 4 demonstrate that in each of the transcripts several nucleotides (denoted by arrows) are methylated by DMS. The distribution of the methylated residues confirms the stem and loop structures generated by computer, as shown in Fig. 3. In a comparison of the reactivities of $A$ and $C$ residues in transcripts from the four infectious variants of PSTVd, i.e., those inciting intermediate to severe symptoms, no obvious differences can be seen in either the upper or the lower portion of the P domain (Figs. 3 and 4).

\section{DISCUSSION}

This report describes the biological effects of several mutations in the VM region of PSTVd. Of the eight CDNAs which were tested for infectivity, four (PSTVdD, PSTVd-M2, PSTVd-M3/D, PSTVd-M3/4) were not infectious. Calculations of the free energy of their most stable secondary structure (Zuker, 1989) showed that, with the exception of PSTVd-M3/4, the introduced mutations dramatically decreased thermodynamic stability (Table 2).

Four of the cDNAs (PSTVd-B5, PSTVd-M1, PSTVd$M 2 / M 1, P S T V d-M 3 / M 1)$ were infectious and resulted in the production of symptoms ranging from intermediate to severe in intensity. The structural properties of these variants were further examined by computer analysis and by limited chemical mapping. As shown in Fig. 3, a comparison of the VM regions of the variants reveals that there is no influence of the mutations on the folding of the right side of the region (which includes PM loop 1), yet there is an effect on the number of bases paired in the stem adjacent to the PM loop 1 . Since the rules of folding adopted in each algorithm used in computer analysis may determine the outcome, one may question the reliability of computer 
A

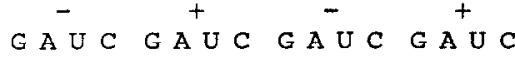

B GÄUC GAUC GA UC GAUC GAUC GAUC

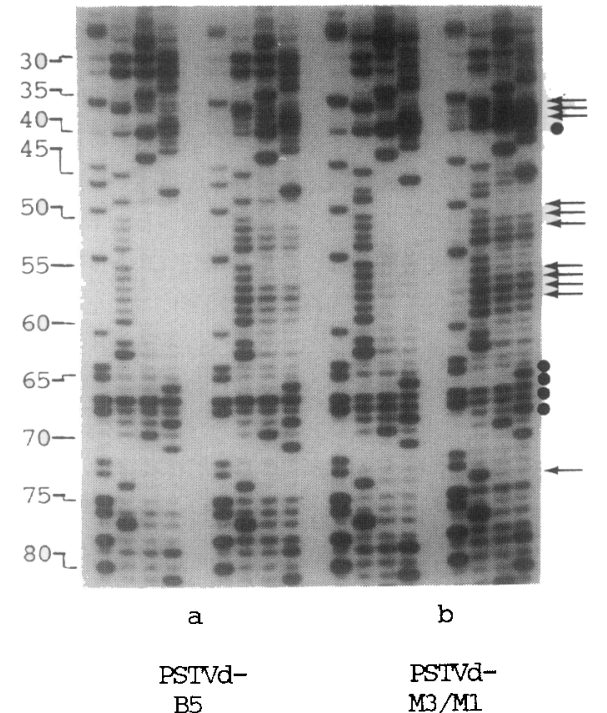

B5

$\mathrm{M} 3 / \mathrm{MI}$

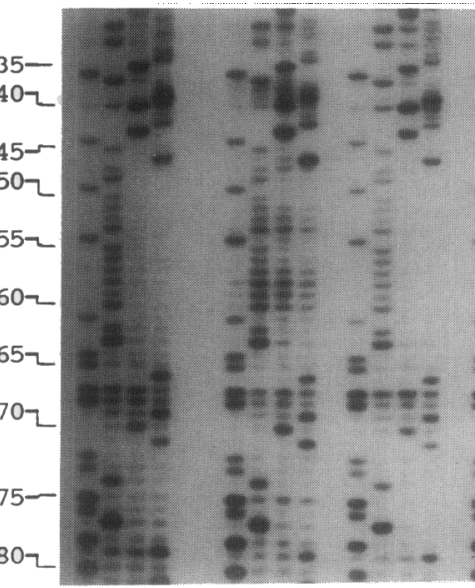

a

PSTVd-

MI

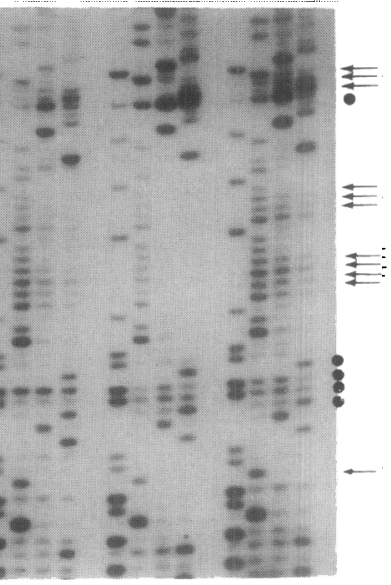

b

PSTVAM2/MI
$\mathrm{C}$

PSTVd-

C
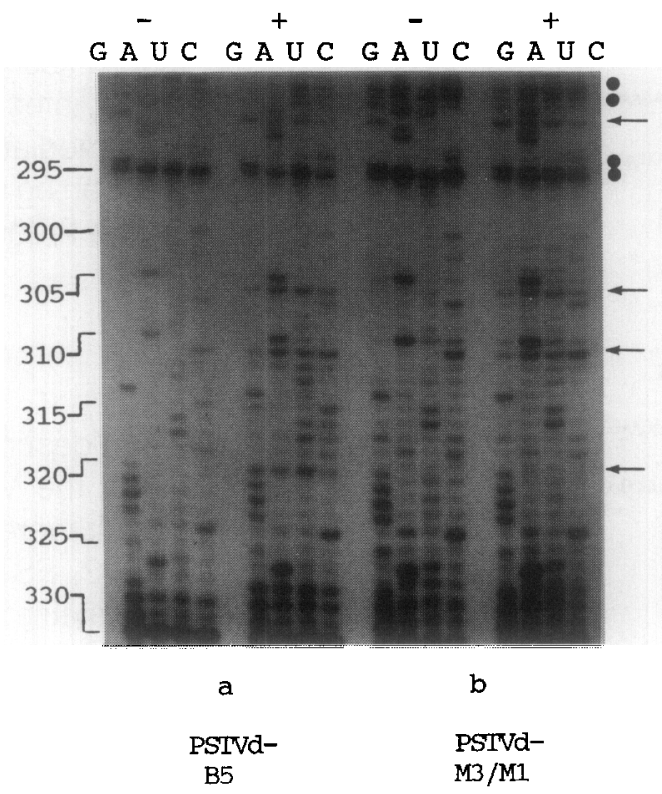

Fig. 4. Detection of modified bases by reverse transcription of RNA templates, as described under Materials and Methods. (Numbers to the left indicate sequence positions in PSTVd.) The RNAs were treated with DMS ( + ) and subjected to reverse transcription using a primer complementary to nucleotides 52-69 of pSP64 (primer 2) (a and b) or nucleotides 340-353 of PSTVd (primer 1) (c) in the presence of dideoxy nucleotides to generate the sequences shown. Unmodified RNAs $(-)$ were sequenced as well and were electrophoresed alongside the modified RNAs as a background for interpretation of the reverse transcription patterns of the modified RNAs. Arrows to the right of the panels indicate the position of modified bases (elongation pauses 1 nucleotide prior to the modified base; Inoue and Cech, 1985). Solid circles indicate strong stops in unmodified transcripts.

analyses alone in predicting the structure of an RNA molecule.

In order to test the predicted models of the variant RNA structures, limited chemical probing experiments were performed. Under the high $\mathrm{Mg}^{2+}$ conditions chosen for the chemical mapping experiments, one might assume that the native structure of the molecule would be rigid (Riesner, 1987). The RNA would assume the most stable conformation and the differences in conformation may be reflected in the pattern of DMS modification. Although metastable bimolecular structures formed by base-pairing of two viroid transcript mole- 
cules in the central conserved region (nt 79-110) may form under the experimental conditions used to fold the molecule (Steger et al., 1986), the conformation of the RNA in the pathogenicity domain should not be affected. The results of chemical mapping demonstrate that, in solution, all of the transcripts of the variant RNAs assume essentially similar conformations to one another.

Although I was able to map the structure of stable helices, it was not possible to experimentally examine the stability of the helices under lower $\mathrm{Mg}^{2+}$ concentrations. The melting of viroid helices is highly cooperative (Steger et al., 1984; Henco et al., 1979) which may explain why no obvious structural differences were detected under the conditions used for denaturing and semidenaturing conditions (data not shown) (Mougel et al., 1987). In fact, the premelting loops which have been theoretically determined for viroids (Steger et al., 1984) have not been accessible to experimental studies becausc their existence is so transicnt. Structural mapping of transcripts, however, can be used as starting point for a more thorough investigation of RNA structure. There may, in addition, be alternative conformations of the RNA which exist in vivo. For example, Branch et al. (1985) found evidence for local tertiary structure in the central conserved region of PSTVd.

The instability calculated for the noninfectious mutants (Table 2) was not reflected in chemical mapping experiments. Investigation of the secondary structure of their corresponding RNA transcripts did not reveal any significant differences in the conformation of the RNA in the VM region compared to PSTVd-B5 (data not shown). Schnölzer et al. (1985) suggest that individual nucleotides in the VM region are not "essential" for replication of PSTVd (based upon observations that this region is highly variable in sequence). The data presented here reveal that artificially introduced single as well as multiple mutations which have not arisen through evolution can abolish infectivity. These mutations may perturb the in vivo secondary structure of the RNA in a way that was not obvious in the chemical mapping experiments. The biological relevance of these observations is unclear and requires further experimental investigation.

Recently Owens et al. (1991) examined the effect of three other distinct mutations in the $V M$ region of PSTVd as part of a more extensive study of the effect of random, chemically produced mutations. Here, too, there was no consistent correlation between basepairing in this region and a reduction in symptom expression. Our combined observations suggest that structural stability alone cannot be the major determinant of pathogenicity and that other factors relating to nucleotide sequence and/or conformation of the RNA must be involved.

It has been postulated that nucleotides within the VM region of PSTVd (specifically PM loop 1) may be important for binding of host factors (Schnölzer et al., 1985). Recent speculation of viroid pathogenesis includes the possible interaction of the lower portion of the $P$ domain in tomato with the $5^{\prime}$ terminus of a host 7 $S$ RNA (Haas et al., 1988). The pathogenic effect of viroids would be the consequence of the formation of a hybrid between the two molecules. This model does not take into account the lack of pathogenicity in closely related species (if $7 \mathrm{~S}$ is highly conserved) nor does it account for mismatches in base-pairing with other viroids which cause severe disease in tomato.

Although viroid-host component complexes have been isolated (Klaff et al., 1989), their host constituents have not been fully characterized. Another possible target in the host cell appears to be related to a dsRNA-dependent protein kinase activity from virus-infected interferon-treated cells. In this case, viroid pathogenicity may involve the differential phosphorylation of host proteins in viroid-infected plants (Hiddinga et al., 1988; Vera and Conejero, 1990). Although circumstantial evidence exists to support both of these theories of viroid pathogenicity, the primary cellular target of viroid interaction with its host is still unknown.

The results presented suggest that a $C$ at residue 317 in the CU bulge loop of PSTVd-M3/M1 may be a contact point for host components and that this interaction may contribute to pathogenicity. Bulged nucleotides have been shown to be important structural motifs in various RNAs (Wu and Uhlenbeck, 1987; White and Draper, 1987). In most cases, a $C$ is present at residue 317 in severe to lethal PSTVd strains, whereas a $U$ is present at this position in mild to intermediate strains (Schnölzer et al., 1985; Zimmat el al., 1990). The presence of a $\mathrm{C}$ or $\mathrm{U}$ may influence protein binding and/or change the relative distance between functional groups elsewhere in the molecule. In a comparison of the infectivity of PSTVd-M2/M1 and PSTVd-M3/M1 (Table 2) it appears that a $C$ at position 317 confers a replicative advantage to the molecule. These results suggest that the early synthesis of viroid RNAs (Table 2) and not the final concentration (Fig. 2B) of thcse RNAs may contribute to early onset and symptom severity of the disease.

Resolution of the different models of viroid secondary structure and their correlation with pathogenicity will require a more rigorous determination of in vivo viroid structure.

\section{ACKNOWLEDGMENTS}

The author acknowledges the skilled technical assistance of Marilyn Hale and Shanta Rishi, helpful discussions with J. Hammond; 
T. O. Diener, and R. A. Owens, and critical review of the manuscript by Drs. E. Tanne, H-T. Hsu, and J. White. This study was partially supported by the USDA Competitive Research Grants Program (Grants 85-CRCR-1738 and 88-37263-3990).

\section{REFERENCES}

Branch, A. D., Benenfeld, B. J., and Robertson, H. D. (1985). Ultraviolet light-induced crosslinking reveals a unique region of local tertiary structure in potato spindle tuber viroid and HeLa 5S RNA. Proc. Natl. Acad. Sci. USA 82, 6590-6594.

FLORES, R. (1984). Is the conformation of viroids involved in their pathogenicity? J. Theor. Biol. 108, 519-527.

HaAs, B., Klanner, A., Ramm, K., and SANGer, H. L. (1988). The $7 S$ RNA from tomato leaf tissue resembles a signal recognition particle RNA and exhibits a remarkable sequence complementarity to viroids. EMBO J. 7, 4063-4074.

Hammond, R. W., Diener, T. O., and OWENS, R. A. (1989). Infectivity of chimeric viroid transcripts reveals the presence of alternative processing sites in potato spindle tuber viroid. Virology $170,486-$ 495.

HAMmOND, R. W., and OWENS, R. A. (1987). Mutational analysis of potato spindle tuber viroid reveals complex relationships between structure and infectivity. Proc. Natl. Acad. Sci. USA 84, 39673971.

Henco, K., SÄnger, H. L., and Riesner, D. (1979). Fine structure melting of viroids as studies by kinetic methods. Nucleic Acids Res. 6, 3041-3059.

Hiddinga, H. J., CRum, C. J., and Roth, D. A. (1988). Viroid-induced phosphorylation of a host protein related to a dsRNA-dependent protein kinase. Science 241, 451-453.

INOUE, T., and CECH, T. R. (1985). Secondary structure of the circular form of the Tetrahymena rRNA intervening sequence: A technique for RNA structure analysis using chemical probes and reverse transcriptase. Proc. Natl. Acad. Sci. USA 82, 648-652.

KEESE, P., and SYMONS, R. H. (1985). Domains in viroids: Evidence of intermolecular RNA rearrangements and their contribution to viroid evolution. Proc. Natl. Acad. Sci. USA 82, 4582-4586.

KLAFF, P., Gruner, R., HeCKer, R., SÄtTler, A., THEISSEN, G., and RIESNER, D. (1989). Reconstituted and cellular viroid protein complexes. J. Gen. Virol. 70, 2257-2270.

Korneluk, R. G., Quan, F., and Gravel, R. A. (1985). Rapid and reliable dideoxy sequencing of double-stranded DNA. Gene 40, 317 323.

Langowski, J., Henco, K., Riesner, D., and Sänger, H. L. (1978). Common structural features of different viroids: Scrial arrange ment of double helical sections and internal loops. Nucleic Acids Res. 5, 1589-1610.

Maniatis, T., Fritsch, E. F., and Sambrook, J. (1982). "Molecular Cloning: A Laboratory Manual." Cold Spring Harbor Laboratory. Cold Spring Harbor, NY.

McGraw, R. A., III (1984). Dideoxy DNA sequencing with end-labeled oligonucleotide primers. Anal. Biochem. 143, 298-303.

Messing, J. (1983). New M13 vectors for cloning. In "Methods in Enzymology" Academic Press, San Diego. (R. Wu, L. Grossman, and K. Moldave, Eds.), Vol. 101, pp. 20-78.

Mougel, M., Eyermann, F., Westhof, E., Romby, P., Expert-BezanCon, A., Ebel, J.-P., Ehresmann, B., and Ehresmann, C. (1987). Binding of Escherichia coli ribosomal protein S8 to 16S rRNA: A model for the interaction and the tertiary structure of the RNA binding site. J. Mol. Biol. 198, 91-107.
OWENS, R. A. (1990). Mutational analysis of viroid pathogenicity: Tomato apical stunt viroid. Mol. Plant Microbe Interact. 3, 374-380.

Owens, R. A., Hammond, R. W., Gardner, R. C., Kiefer, M. C., THOMPSON, S. M., and CRESS, D. E. (1986). Site-specific mutagenesis of potato spindle tuber viroid CDNA: Alterations within premelting region 2 that abolish infectivity. Plant Mol. Biol. 6,179 192.

OWENS, R. A., THOMPson, S. M., and Steger, G., (1991). Effects of random mutagenesis upon potato spindle tuber viroid replication and symptom expression. Virology 185, 18-31.

RIESNER, D. (1987). Physical chemical properties: Structure formation. In "The Viroids" (T. O. Diener, Fd.), pp. 63-98. Plenum, NY.

SÄNGER, H. L. (1982). Biology, structure, functions and possible origin of viroids. In "Encyclopedia of Plant Physiology New Series'" (B. Parthian and D. Boulter, Eds.), Vol. 14B, pp. 368-454. Springer-Verlag, Berlin.

Schinölzer, M., HaAs, B., Ramm, K., Hofmann, H., and Sänger, H. L. (1985). Correlation between structure and pathogenicity of potato spindle tuber viroid (PSTV). EMBO J. 4, 2181-2190.

SHIKATA, E. (1990). New viroids from Japan. Semin. Virol. 1, 107115.

Steger, G., Hoffmann, H., Förtsch, J., Gross, H. J., Randles, J. W., SÄNGER, H. L., and RIESNER, D. (1984). Conformational transitions in viroids and virusoids: Comparison of results from energy minimization algorithm and from experimental data. J. Biomol. Struct. Dyn. 2, 543-571.

Steger, G., Tabler, M., Brüggemann, W., Colpan, M., Klotz, G., SÄNGER, H. L., and RIESNER, D. (1986). Structure of viroid replicative intermediates: Physico-chemical studies on SP6 transcripts of cloned oligomeric potato spindle tuber viroid. Nucleic Acid's Res. 14, 9613-9630.

TABLER, M., and SÄNGER, H. L. (1984). Cloned single- and doublestranded DNA copies of potato spindle tuber viroid (PSTV) RNA and co-inoculated DNA fragments are infectious. EMBO J. 3, 3055-3062.

Vera, P., and CONEJeRo, V. (1990). Citrus exocortis viroid infection alters the in vitro pattern of protein phosphorylation of tomato leaf proteins. Mol. Plant Microbe Interact. 3, 28-32.

VISVADER, J. E., and SYMONS, R. H. (1985). Eleven new sequence variants of citrus exocortis viroid and the correlation of sequence with pathogenicity. Nucleic Acids Res. 13, 2907-79?0.

VISVADER, J. E., and SYMONS, R. H. (1986). Replication of in vitro constructed viroid mutants: Location of the pathogenicity-modulating domain of citrus exocortis viroid. EMBO J. 5, 2051-2055.

WHITE, S. A., and DRAPER, D. E. (1987). Single base bulges in small RNA hairpins enhance ethidium bromide binding and promote an allosteric transition. Nucleic Acids Res. 15, 4049-4064.

WU, H.-N., and UHLENBECK, O. C. (1987). Role of a bulged A residue in a specific RNA-protein interaction. Biochemistry 26, 8221 8227.

Zimmat, R., Gruner, R., Hecker, R., Steger, G., and Riesner, D. (1990). Analysis of mutations in viroid RNA by non-denaturing and temperature-gradient gel electrophoresis. In "Proceedings of the Sixth Conversation in Biomolecular Stereodynamics" (R. Sarma and M. Sarma, Eds.), Vol. 3, pp. 339-357. Adenine Press.

ZIMMERN, D., and KAESBERG, P. (1978). 3'-Terminal nucleotide sequence of encephalomyocarditis virus RNA determined by reverse transcriptase and chain-terminating inhibitors. Proc. Natl. Acad. Sci. USA 75, 4257-4261.

ZUKER, M. (1989). On finding all suboptimal foldings of an RNA molecule. Science $244,48-52$. 